\section{Impact of Early Passive Exercise With Cycle Ergometer on Ventilator Interaction}

\section{To the Editor:}

We read and appreciate the interesting article entitled "Impact of Early Passive Exercise With Cycle Ergometer on Ventilator Interaction" by Silva et al. ${ }^{1}$ The study aimed to assess the patient-ventilator interaction during passive mobilization performed with a cycle ergometer. This study included deeply sedated individuals who were mechanically ventilated for $<72 \mathrm{~h}$. Parameters such as the asynchrony index and the types of asynchrony were evaluated for $20 \mathrm{~min}$, followed by 10 min of recovery.

This issue is extremely relevant because patient-ventilator asynchrony is associated with worse clinical outcomes such as reintubation, increased length of ICU and hospital stay, and mortality., 2,3 Thus, we have some concerns regarding the study.

First, the sample was composed of 113 patients, and only 8 subjects were included. Despite the exclusion criteria, most individuals were not submitted to the passive mobilization protocol due to hemodynamic instability (ie, 45 patients). The authors described that the ICU was a clinicalsurgical unit and used severity scales to characterize the sample (Sequential Organ Failure Assessment and Simplified Acute Physiology Score II); however, they described a significant number of exclusions in a study that was conducted over approximately 2 years. Thus, we believe that there is a need to characterize the enrolled subjects better. In this sense, we also believe that a small sample and the lack of sample size calculation can hardly infer results on the impact

Second, although the authors used the Richmond Agitation-Sedation Scale, the variability allowed (ie, -3 to -5 ) associated with a small sample size may also interfere with both the results and the occurrence of asynchrony. Furthermore, it would be essential to standardize the analgesia MSc, R. Professora Maria Flora Pausewang, CEP 88036-800, Florianópolis, Brazil. E-mail: fisiocattelan@gmail.com.

The authors have disclosed no conflicts of interest. of mobilization on ventilatory asynchrony.

Correspondence: Kelly Cattelan Bonorino

assessment to exclude the possibility of asynchrony caused by pain or discomfort. The pain assessment and monitoring using appropriate scales (eg, Critical care pain observation tool or Behavioral pain scale) ${ }^{4}$ would also be essential to comprehend whether asynchrony was due to pain or discomfort during mobilization.

Third, only one (blinded) researcher evaluated asynchrony. It would be interesting to have more than one evaluator and compare the results to exclude bias. The sensitivity and positive predictive values of analyzing breath-by-breath waveforms are low ( $22 \%$ and $32 \%$, respectively), and trained professionals recognize less than one third of asynchronies. It is also important to highlight that clinicians' ability to identify asynchronies visually is influenced by their expertise and the type of asynchrony. ${ }^{5}$

Although the types of asynchrony assessed were described in the methodology, the authors did not include the reverse-triggering, a common asynchrony found in deeply sedated patients, ${ }^{6-9}$ and this type of asynchrony can be confounded with insufficient flow. Su et $\mathrm{al}^{8}$ observed that $30 \%$ of their subjects presented reverse-triggering in the late inspiratory and early expiratory phases.

The most frequent asynchronies observed during the passive exercise were ineffective triggering and insufficient flow. An inspiratory-flow mismatch is more common in constant modes such as volume controlled continuous mandatory ventilation ${ }^{10}$; however, in this study, all subjects received pressure controlled ventilation.

Therefore, the authors need to supplement the information concerning the admitted subjects' characterization, which probably justifies the large number of excluded patients. We would like to know whether the authors calculated the sample size of this study. It is important to clarify the details of the pain monitoring to comprehend whether asynchrony was due to pain or discomfort during mobilization. It is also essential to clarify why only one reviewer evaluated asynchrony and why reverse-triggering was not added to the evaluation.

Kelly C Bonorino
Intensive Care Unit
Polydoro Ernani de São Thiago Hospital
Universidade Federal de Santa Catarina
Florianópolis, Brazil

Kelly C Bonorino Intensive Care Unit

DOI: $10.4187 /$ respcare.08643
Katerine C Cani

Physiotherapy Department

Centro de Ciências da Saúde e do Esporte

Universidade do Estado de Santa Catarina

Florianópolis, Brazil

\section{REFERENCES}

1. Silva JR, Reboredo MM, Bergamini BC, Netto CB, Vieira RS, Pinto SP, et al. Impact of early passive exercise with cycle ergometer on ventilator interaction. Respir Care 2020;65(10):1547-1554.

2. Esperanza JA, Sarlabous L, de Haro C, Magrans R, Lopez-Aguilar J, Blanch L. Monitoring asynchrony during invasive mechanical ventilation. Respir Care 2020;65 (6):847-869.

3. Blanch L, Villagra A, Sales B, Montanya J, Lucangelo U, Lujan M, et al. Asynchronies during mechanical ventilation are associated with mortality. Intensive Care Med 2015;41 (4):633-641.

4. Kotfis K, Zegan-Barańska M, Szydłowski Ł, Żukowski M, Ely EW. Methods of pain assessment in adult intensive care unit patients - Polish version of the CPOT (Critical Care Pain Observation Tool) and BPS (Behavioral Pain Scale). Anaesthesiol Intensive Ther 2017;49 (1):66-72.

5. Chacón E, Estruga A, Murias G, Sales B, Montanya J, Lucangelo U, et al. Nurses' detection of ineffective inspiratory efforts during mechanical ventilation. Am J Crit Care 2012;21(4):e89-e93.

6. Akoumianaki E, Lyazidi A, Rey N, Matamis D, Perez-Martinez N, Giraud R, et al. Mechanical ventilation-induced reverse-triggered breaths: a frequently unrecognized form of neuromechanical coupling. Chest 2013;143(4):927-938

7. Murias G, de Haro C, Blanch L. Does this ventilated patient have asynchronies? Recognizing reverse triggering and entrainment at the bedside. Intensive Care Med 2016;42(6):1058-1061.

8. Su HK, Loring SH, Talmor D, Baedorf Kassis E. Reverse triggering with breath stacking during mechanical ventilation results in large tidal volumes and transpulmonary pressure swings. Intensive Care Med 2019;45(8):1161-1162.

9. Yoshida T, Nakamura MAM, Morais CCA, Amato MBP, Kavanagh BP. Reverse triggering causes an injurious inflation pattern during mechanical ventilation. Am J Respir Crit Care Med 2018;198(8):1096-1099.

10. Murias G, Lucangelo U, Blanch L. Patientventilator asynchrony. Curr Opin Crit Care 2016;22(1):53-59. 